\title{
Face Recognition Based on Viola-Jones Face Detection Method and Local Binary Pattern (LBP)
}

\author{
Suhad Ibrahim Mohammed \\ Suhad698@gmail.com
}

\begin{abstract}
- in this paper, the system consists of many steps, the first step includes the histogram equalization, detection, feature extraction, and classification. At first, the data set of a face image is segmented into four segments, after that Local Binary Pattern (LBP) algorithm is performed to extract features for each segment. The best feature vectors for all persons are stored in a new dataset in the next stage in order to be used in the testing phase. Finally, the accuracy rate of performance is evaluated to prove its robustness. Experiments show satisfying results and more accuracy achieved by the paper.
\end{abstract}

Index Terms - Face image Recognition; LBP, Viola-Jones; MUCT, Feature Extraction.

\section{INTRODUCTION}

The face area is important and it has attracted the attention in social communications, playing a major part in transferring sentiment and identity. Identifying faces by seeing them in our lifetime enables us to identify known faces immediately even after years of divergence [1][2].

Face detection is an instance of advanced aim detection [3]. The process is affected by several factors such as shape, indexes of refraction, position, obstruction and enlightenment. A human face is a very complex object with characters that can vary from time to time, sometimes very quickly. The face is an extremely distortion object, and facial expressions enter a wide assortment of possible configurations. Time-varying changes contain growth and removing facial expression. It should be very clear that the person face is more difficult than other objects due to model and recognition [4].

\section{RELATED WORK}

Many researchers have attempted to find the results of the problem of face recognition; something that even little boys can do so effectively, simply and obviously, from that time they may be born. Scientists and engineers were anxious with increasing face image recognition systems.

- Sinha C., 2013 [8] investigated face images of gender classification applying, SVM and PCA. The researcher initially intended to introduce a skin color segmentation method using an extracted face image area. The face image area was then reduced feature extraction by applying PCA, which was encoded in a second class by statistics of data. Those PCA were supplied as information to SVM for classification.

- Yuan L., et al., (2013) [9] introduced more versatile techniques in face recognition during a preprocessing stage of the image by adopting eight eyes segmentation, geometry normalization and energy normalization. Then to attract the global features of the complete expression image as well as the local texture feature of mouth, they used Local Binary Pattern (LBP) and PCA. After the two kinds of feature ultimate recognition and expression classification, the SVM algorithm was used. For the experiment, from the total samples of training images, half of the images had poor condition of illumination while the other half had clear condition. Seven recognition expressions 
were involved in an experiment out of six expressions (happy, surprise, sadness, anger, fear, and disgust) and the result of the average recognition rate was $94 \%$.

- Di Huang, et al., 2014[10] used the LBP method in circumstances of face image recognition. Various characters were discussed, containing modern evolution of LBP, LBP feature extraction, the applications in various face image analysis tasks and existing systems.

- Reem Ibrahim 2015[11] proposed a classification method. First, the method was implemented to reduce different lighting effects by applying the histogram equalization. The segmentation is made by applying Viola and Jones for face image detection to reduce the image size of the face part of the image, which eliminates the unwanted region from the image.

\section{FEATURE EXTRACTION BY USING LBP ALGORITHM}

The LBP method labels the pixel by thresholding a $4 \times 4$ or $3 \times 3$ neighborhood of a face image for each pixel with the center number such as a binary number of that equivalent decimal number applied for labeling. Local Binary Pattern is used to derive binary numbers.

The restriction of LBP method is the deal with the operator that was later increased to apply neighborhoods of various sizes, the texture at different scales, so that it is small $3 \times 3$ or $4 \times 4$ neighborhood, which cannot capture the dominant features with huge scale constructions [12]. The local neighborhood is defined as a set of the example points that do not fall in the pixels that are displayed applying bilinear interpolation and pattern points equally spaced on a circle centered at the pixel to be labeled. Figure 1 shows a sample of the LBP method.

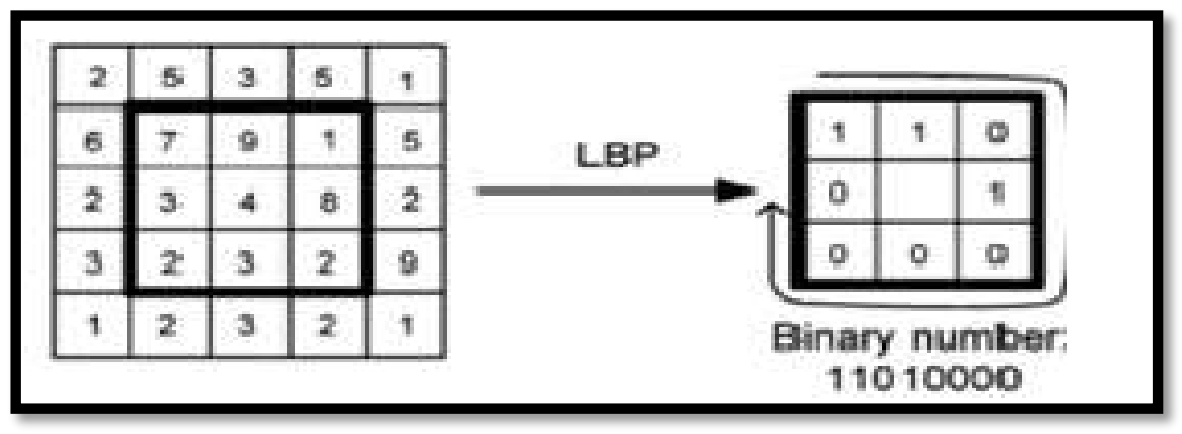

FIG. 1. A SAMPLE OF THE LBP OPERATOR

The MUCT database has 240 faces; these databases differ in scope and size. The individuals in this database are high school teachers, parents, university students and employees [13]. Figure 2 depicts the MUCT database for images.

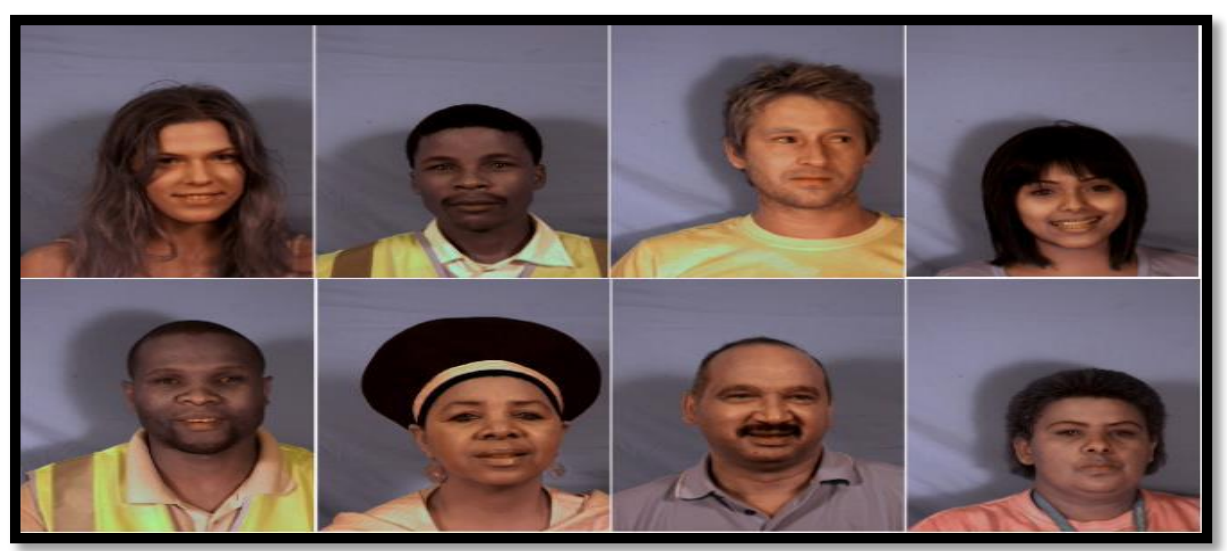

Fig.2. IMAGES OF MUCT DATABASE 


\section{FACE DETECTION}

The Viola and Jones is the first method in 2001 to present face image detection at extremely rapid rates [14]. It is the scheme for correct and fast face detection by the application of adaboost machine learning. There are three features of Viola-Jones algorithm which are:

\section{Integral image}

It needs to compute an integral image acquired by doing few operations per pixel. In addition, it needs the computation of Haar features so that it needs fast detection of objects. Behind this computation, in constant time, Haar features of any sample can be computed.

\section{The adaboost learning algorithm}

Learning must exclude a common of features probable in the image for fast classification. Critical features are extracted by this algorithm while discarding all other unnecessary features. It created flexible classifiers from the amount of extracting related features.

\section{The cascade classifier}

It discards the background and concentrates on objects such as parts. Cascade is a sort of mechanism that rejects areas that are not likely to include any object and the method knows its region of interest. This is very fast in face detection [15]. The cascade classifier is shown in Figure 3.

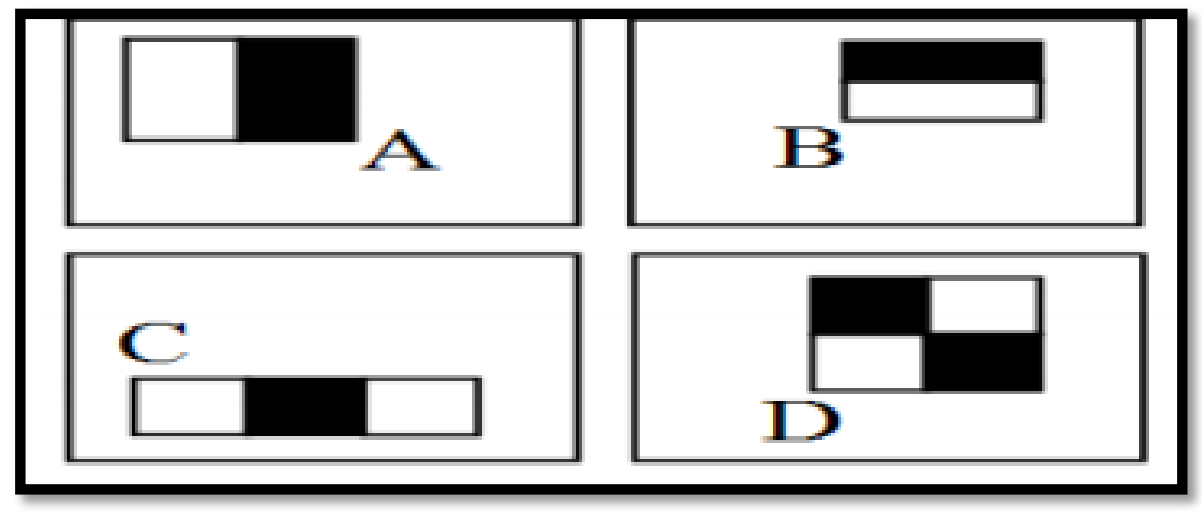

FIG. 3. THE CASCADE CLASSIFIER

\section{THE PROPOSED SYSTEM}

The system consists of training and testing phases as illustrated in Figure 4, which shows the proposed face image recognition. In the training phase, several algorithms have been used to create a data set which will be used in the testing phase to recognize the right faces, the training phase is based on the following steps:

- The preprocessing image step: It is used to enhance the input images by converting the image color to grayscale and applying the histogram equalization.

- Faces Detection step: It uses the Viola - Jones to detect single or multi-face in each input image.

- Features extraction step: Features vector in this step will be extracted based on the Local Binary Pattern (LBP) algorithm. Fig. 4 shows sample images paper.

- The experimental results of the system are obtained by using the algorithms which are explained previously for each step used for the system and based on the image dataset, which contains 240 images.

- The images in the dataset are taken in different circumstances, for this reason the histogram equalization was used to show the detail of the used images, to detect face image properly and to enhance the image quality. 


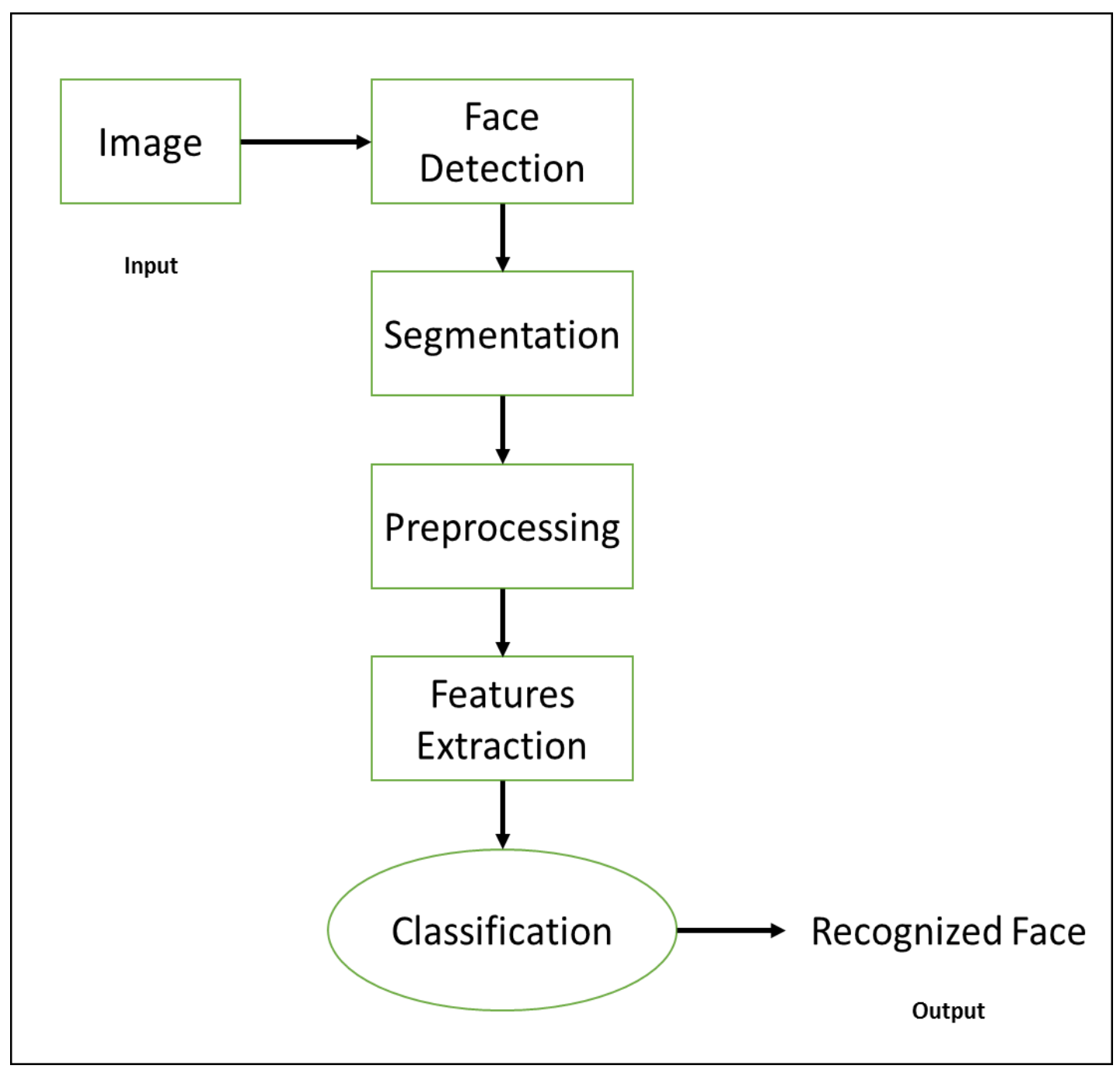

Fig. 4. A Diagram of the Proposed System

\section{A. Detection Step}

In this step, 240 images of 20 persons have used 12 images for each person and these images, which are taken in different environments, are used to detect four segments from each image of the persons which are: eyes, mouth, nose, and face. Figure 4. shows the faces detection for four segments.

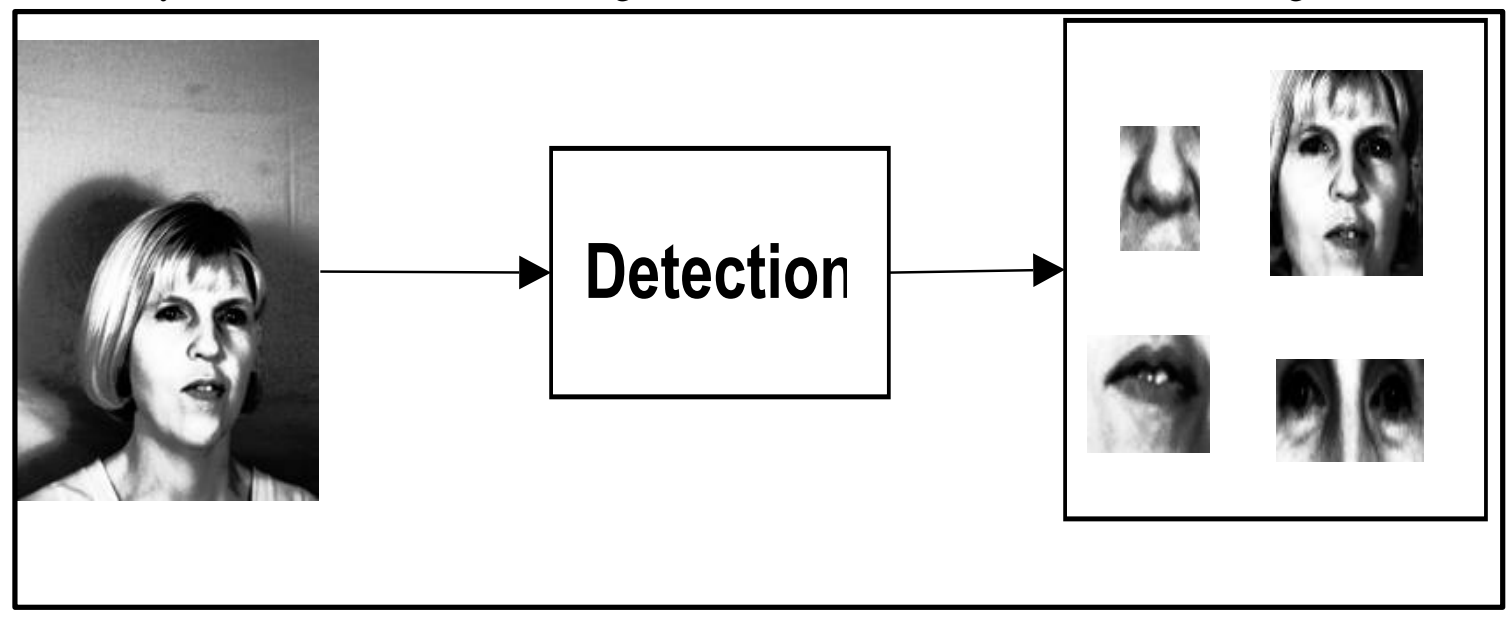

Fig.5. Detection of Four SEGMENTS 


\section{B. FEATURE EXTRACTION}

The feature vector is a 2-dimensional vector of a mathematical operator that represents the features of face image recognition. It was used to reduce the dimensionality of the feature. The sets of features of the eyes, mouth, face, and nose regions were extracted and selected by using the LBP algorithm. Feature extraction includes decreasing the amount of means required to explain a huge number of features in the study. The LBP has been applied including dimension reduction and feature extraction technique from the original face images.

\section{LBP Approach}

The LBP approach may be planned by applying its statistical dispersion to give local texture description and to regionalize an image. In addition, the approach extracts and summarizes their distribution, and then trains features.

- For each pixel in a cell, compare the pixel to each of its 8 neighbors .

- In the above step, the neighbors considered can be changed by varying the radius of the circle around the pixel, $\mathrm{R}$ and the quantization $\mathrm{F}$ the angular space $\mathrm{P}$.

- When the center pixel's value is greater than the neighbor's value, write "0". Otherwise, write "1". This gives an 8-digit binary number.

- Compute the histogram, over the cell, of the frequency of each "number" occurring.

- Optionally normalize the histogram.

\section{RESULTS}

The face image recognition applies the LBP method to recognize orientations, different lighting conditions, and different face images. The database was used in our experiments for each individual with few variations.

In this paper, the result used three images of 20 persons and applied six segments types of eyes, mouth, nose, eyes with a nose, eyes with nose and face segments, in training images 60 of each segment, and testing phase segments 60 from dataset 240 images of 20 persons of each person 12 images.

In this paper, the result of performance accuracy of the eyes segment is $87.67 \%$, the mouth segment is $88.87 \%$, nose segment is $55.56 \%$, eyes with the nose is $34.67 \%$, eyes with a mouth is $45.56 \%$ and face $100 \%$ accuracy rate performance of each segment, as displayed in Table(1).

TABLE I: THE FACE IMAGE RECOGNITION RATE OF LBP

\begin{tabular}{ccccc}
\hline Number & Type sample & Training sample & Testing Sample & Evaluation (\%) \\
\hline 1 & Eye & 60 & 60 & 87,67 \\
2 & Mouth & 60 & 60 & 88.87 \\
3 & Nose & 60 & 60 & 55.56 \\
4 & Eye and nose & 60 & 60 & 34.67 \\
5 & Eye and mouth & 60 & 60 & 45.56 \\
6 & Face & 60 & 60 & 100 \\
\hline
\end{tabular}

The result used 12 images of 20 persons and applied six segments types of eyes, mouth, nose, eyes with nose, eyes with nose and face segments, in training images 240 of each segments, and testing phase segments 240 from dataset 240 images of 20 person of each person 12 images .

In this paper, the result of performance accuracy of eyes segment is $98.75 \%$, mouth segment is $52.7 \%$, nose segment is $46.7 \%$, eyes with nose is $70.34 \%$, eyes with mouth is $65.56 \%$ and face is $98.7 \%$ accuracy rate performance of each segment, as displayed in Table (2). 
TABLE 2: THE FACE IMAGE RECOGNITION RATE OF LBP

\begin{tabular}{ccccc}
\hline Number & Type sample & Training sample & Testing sample & Evaluation (\%) \\
\hline 1 & Eye & 240 & 240 & 98.75 \\
2 & Mouth & 240 & 240 & 52.7 \\
3 & Nose & 240 & 240 & 46.7 \\
4 & Eye and nose & 240 & 240 & 70.34 \\
5 & Eye and mouth & 240 & 240 & 67.56 \\
6 & Face & 240 & 240 & 98.7 \\
\hline
\end{tabular}

\section{CONCLUSION}

The MUCT dataset is taken in different circumstances for training and evaluating a wide assortment of models. A face detection algorithm was introduced and implemented, based on LBP features, utilizing another feature which is computationally simpler than Haar-like feature, motivated by the fact that computing Haar-like features is too computationally intensive for face image. Although LBP feature is simpler, the implementation shows that it is enough to discriminate faces and non faces faster.

\section{REFERENCE}

[1] A. F. Mahdi, "face recognition system based on artificial neural network," Iraqi Comm Comput Informatics Informatics Inst Postgrad Stud.

[2] M. Rahim, , et al., "Face Recognition using Local Binary Patterns (LBP)" in Global Journal of Computer Science and Technology Graphics \& Vision, vol. 13 Issue. 4, 2013.

[3] A. Harsoyo , et al.," Design of Face Recognition system using Local Binary Pattern and Clahe on Smart Meeting Room System" in IEEE 3rd International Conference on System Engineering and Technology, Malaysia, 2013.

[4] W. Zhao, R., et al., Face recognition: A literature survey, “ACM Computing Surveys (CSUR)”, December 2003.

[5] A. Harsoyo, M.C, et al.," Design of Face Recognition system using Local Binary Pattern and Clahe on Smart Meeting Room System" in IEEE 3rd International Conference on System Engineering and Technology, Malaysia, 2013.

[6] K. Meena and Dr. A. Suruliandi, "Local Binary Patterns and its Variants for Face recognition” in IEEE International Conference on Recent Trends in Information Technology, Chennai, June 3-5, 2011.

[7] M. Barnard, , et al., "Audio-Visual Face Detection for Tracking in a Meeting Room" in 16th International Conference on Information Fusion,Instanbul, Turkey, July 9-12, 2013.

[8] Singh, Ranjeet, and Mandeep Kaur. "Face Recognition and Detection using Viola-Jones and Cross Correlation Method . 2013.

[9] Yuan L., et al, Face Recognition in Digital Video by Using Artificial Intelligence Techniques, University of Technology- Department of Computer Sciences., 2013.

[10] Di Huang, et al. , Facial Image Analysis Based on Local Binary Patterns , to the IEEE for possible publication, 2014.

[11] Reem Majeed Ibrahim, Classification of Face Image Based on Gender Using Intelligent Method, Ministry of Higher Education and Scientific Research University of Technology Department of Computer Sciences, October 2015.

[12] Ahonen, Timo, et al.,. "Face description with local binary patterns: Application to face recognition." IEEE transactions on pattern analysis and machine intelligence 28.12 (2006): 2037-2041.

[13] Milborrow, Stephen, et al., "The MUCT landmarked face database." Pattern Recognition Association of South Africa 201.0 (2010)

[14] Viola, Paul, and Michael J. Jones. "Robust real-time face detection." International journal of computer vision 57.2 (2004): 137-154.

[15] Jones, Michael, and Paul Viola. "Fast multi-view face detection." Mitsubishi Electric Research Lab TR-20003963.14 (2003): 2. 